\title{
Ketamine - A Multifaceted Drug
}

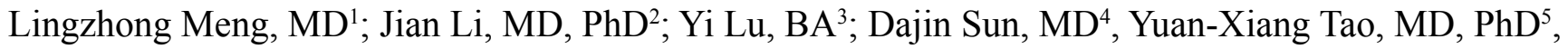
Renyu Liu, MD, $\mathrm{PhD}^{6}$, Jin Jun Luo, $\mathrm{MD}, \mathrm{PhD}^{7}$

${ }^{1}$ Department of Anesthesia and Perioperative Care, University of California San Francisco; ${ }^{2}$ The $1{ }^{\text {st }}$ Affiliated Hospital of Soochow University, China; ${ }^{3}$ School of Medicine, University of California San Francisco; ${ }^{4}$ Department of Anesthesiology, Renji Hospital, Jiaotong University School of Medicine; ${ }^{5}$ Department of Anesthesiology, Rutgers New Jersey Medical School, The State University of New Jersey; ${ }^{6}$ Department of Anesthesiology and Critical Care, Perelman School of Medicine at the University of Pennsylvania; ${ }^{7}$ Departments of Neurology and Pharmacology, Temple University

There is a petition for tight control of ketamine from the Chinese government to classify ketamine as a Schedule I drug, which is defined as a drug with no currently accepted medical use but a high potential for abuse. However, ketamine has unique properties that can benefit different patient populations. Scholars from the Translational Perioperative and Pain Medicine and the International Chinese Academy of Anesthesiology WeChat groups had an interactive discussion on ketamine, including its current medical applications, future research priorities, and benefits versus risks. The discussion is summarized in this manuscript with some minor edits.

\section{Lingzhong Meng (University of California San} Francisco, USA): Dr. Gelb from the University of California San Francisco (UCSF) told me today that the Chinese government is trying to make ketamine a Schedule I drug. This move is a severe setback for a drug that has unique properties that can benefit patients suffering from chronic pain and depression, to name a few conditions. This move will significantly restrict the drug, which does not have a suitable substitute, for clinical use. Please join Dr. Gelb and me in trying to convince the government from removing this drug from our armamentarium for patients who depend on it. Thank you for your support! (Schedule I drugs, substances, or chemicals are defined as drugs with no currently accepted medical use and a high potential for abuse. Schedule I consists of the most dangerous drugs of all of the drug schedules with po- tentially severe addictive properties.)

Daqing Ma (Imperial College London, UK): Are there any data showing ketamine's abuse in China or the United States?

Jie Zhou (Brigham Women's Hospital, USA): Yes, ketamine is the number one illicit drug in China, including Hong Kong. Ketamine is less popular in the United States.

\section{Adrian Gelb (University of California San Fran-} cisco, USA): We have been in regular contact with Dr. Lize Xiong (Xijing Hospital, China) and Dr. Yuguang Huang (Peking Union Medical College Hospital, China) about this ketamine issue. Yuguang did meet with some government officials and his report was similar to yours. The information we have is that it is probable that the United States is going to propose categorizing ketamine as a Schedule II drug (it is currently a Schedule III). This is better than Schedule I but will still place some obstructions on the use of the drug in many countries. When morphine was scheduled in the 1980 s, the experience was that many countries such as India required so many licenses and imposed such severe penalties that companies refused to import the drug. As a result, morphine is hard to obtain for medical use in India - a company needs 6 licenses to make it and the penalty for an expired license is 20 years in jail. 
Jie Zhou: The WHO report stated that ketamine is illegally shipped to the United States via Mexico.

Categorizing ketamine in schedule II is a better choice and shows a better gesture from the US side.

\section{Liming Zhang (University of Pittsburgh, USA):}

Drug K (ketamine) powder is fairly common in entertaining venues in China. It is involved in many criminal cases and is also known as a "rape drug". When I talked to a pharmaceutical company about the development and production of ketamine several years ago, it was immediately associated with a drug of abuse rather than an analgesic. Thus, ketamine abuse is a major issue and its manufacture requires licensing. I have only seen bottled liquid ketamine for medical use in the United States. However, ketamine powder can be inhaled or mixed in liquids in entertainment venues in China.

Renyu Liu (University of Pennsylvania, USA): Ketamine is a drug of choice in the perioperative period for patients who are on chronic opioids.

\section{Haobo Ma (Beth Israel Deaconess Medical Center,}

USA): In my hospital, chronic pain patients who are not well-controlled with narcotics are usually put on a ketamine infusion. The three levels of ketamine infusion are $0.1-0.3 \mathrm{mg} / \mathrm{kg} / \mathrm{hr}, 0.3-0.5 \mathrm{mg} / \mathrm{kg} / \mathrm{hr}$, and $0.5-1$ $\mathrm{mg} / \mathrm{kg} / \mathrm{hr}$. Postoperative ketamine infusion requires the involvement of the acute pain service and can also be given as a bolus. I usually give a small bolus of 20 mg to titrate to effect. In the bariatric surgery patient population, researchers have also studied infusing ketamine to reduce the use of opioids. Ketamine is not inferior to opioids, and is associated with less postoperative nausea, vomiting, and respiratory depression. One side effect, as we know, is hallucination in some patients, although this is less common with low dose infusions $(<2.5 \mathrm{mcg} / \mathrm{kg} / \mathrm{min})$. Krystal JH published two papers in 1994 and 1998 defining this rate as equivalent to $0.15 \mathrm{mg} / \mathrm{kg} / \mathrm{hr}[1,2]$.

Xiang Qian (Stanford University, USA): We should follow up on our discussion of ketamine's legal status in China. In the United States, ketamine has a bright future for treating refractory pain conditions such as complex regional pain syndrome, failed back surgery syndrome, cancer pain, acute surgical pain, and is now increasingly used in palliative care, hospital, and home care settings with solid supporting data.

Transl Perioper \& Pain Med 2015; 1(2)
Gang Li (Private Practice, USA): For acute pain refractory to conventional modalities, ketamine is a good option. What do you think about its efficacy and long-term benefits on chronic refractory non-cancer pain?

Xiang Qian: Exactly. One of the goals of using ketamine is to reduce opioid use, which can cause nausea, vomiting, and ileus, and is one of the top reasons for hospital readmissions after surgery. At this time, the best evidence for the use of ketamine in pain control is still limited to the acute pain setting; the data on chronic pain is controversial, especially for oral ketamine, which has very poor bioavailability.

Lingzhong Meng: Previous studies showed that ketamine has cancer-promoting properties [3-5]. For cancer patients, how do we weigh ketamine's impact on survival even though it helps with pain? Survival may be an indicator of the overall effects of ketamine that are the net result of the advantages and disadvantages.

\section{Weidong Gao (Johns Hopkins University, USA):}

At last night's Anesthesia \& Analgesia editorial board meeting, it was mentioned that a paper on ketamine should be considered for publication in the journal to support the use of ketamine in light of the Chinese government's tightening control of the drug.

Gang Li: High-dose opiate therapy can cause immune suppression and, in theory, promote cancer [6,7]. The same is true with inadequate pain control in cancer patients.

\section{Yuanxiang Tao (Rutgers New Jersey Medical}

School, USA): This is why the co-administration of ketamine may reduce the dose of opioids needed and its associated side effects. How about the co-administration of ketamine and opioids in neuropathic pain?

\section{Vivian Bi (University of Mississippi, USA): What} is the reason for ketamine's poor bioavailability? Is it due to metabolism or poor solubility?

Gang Li: Ketamine is broken down by bile acids and therefore only has $20 \%$ bioavailability when given orally.

Vivian Bi: If it can be stabilized, would there be a significantly higher demand for oral ketamine?

Page 21 
Gang Li: There would, but doing so increases the risks and concerns for misuse and abuse.

Xiang Qian: There is a nasal spray form available already.

Weidong Gao: Have any of you considered writing a review on ketamine? Why not round up some interested people to work on it? It would be a well-received article. It is even better to get someone in China involved.

Renyu Liu: This is a great suggestion. We are planning a special issue/event for pharmacology and translational medicine using a collaborative model involving experts from China and the United States. I encourage those who have funding, especially NIH funding, to participate in this effort to write reviews related to this topic. Please contact me if you are in the United States or England, and please contact Dr. Yuguang Huang if you are in China.

Lingzhong Meng: How about this name - The bogeyman ketamine: its tricks and treats in cancer patients.

Xiqing Cao (Medstar Washington Hospital Center, USA): Ketamine is no longer rarely used. We use it routinely in patients with chronic pain syndrome, total knee arthroplasty, bariatric surgery, obstructive sleep apnea, and burns as part of a multimodal approach. We also sometimes use it during cesarean sections.

Lingzhong Meng: These are non-cancer scenarios. For example, ketamine has been shown in meta-analyses to be superior to placebo in the treatment of unipolar depression that was refractory to other modalities $[8,9]$. In cancer patients, ketamine helps with cancer pain but may promote cancer growth. What is the long-term effect of ketamine use in the cancer patient population? It seems that we do not know if ketamine improves the survival of cancer patients.

$\underline{\text { Xiqing Cao: }}$ : Excellent question. We need to know long-term outcomes. Do you know if ketamine has any negative effects on the immune system?

Lingzhong Meng: It suppresses natural killer cell activity, which is the first line of cancer defense [3-5].

$\underline{\text { Xiqing Cao: }}$ I remember that opioids suppress more natural killer cell activity.

Yuanxiang Tao: Is there any published evidence? How does ketamine affect immune cells?

Weifeng Tu (Guangzhou Military General Hospital, China): Does its immunosuppressive effects relate to drug dose and/or the frequency of administration?

Xiqing Cao: Personally I like to use epidural anesthesia with local anesthetic agents for cancer patients. However, not every surgeon agrees.

Lingzhong Meng: There are some animal studies examining ketamine's effect on immunity.

Yuanxiang Tao: Are there cytotoxic effects?

Lingzhong Meng: Ketamine suppresses natural killer cell activity [1-3].

Xiqing Ca0: This is why Dr. Sessler (Cleveland Clinics, USA) is conducting multicenter clinical trials comparing regional anesthesia with intravenous patient-controlled analgesia. We need similar studies to prove our hypothesis on ketamine. This is a very interesting discussion.

Jin Jun Luo: I finally finished reading the opinions of every expert. I learned a lot, thanks. Based on my personal clinical experience, ketamine has a short-term effect on pain control in patients with chronic pain, such as reflex sympathetic dystrophy, chronic regional pain type 1, and chronic neuropathic pain. Ketamine is very costly when given intravenously in the ICU. Usually the pain returns by the time the patients are at the hospital's door at discharge or within a few days. I strongly discourage using ketamine for patients with chronic pain as it is not cost-effective.

Xiulu Ruan (Private Practice, USA): I agree. Just like rapid detoxification, it is a way to generate a lot of revenue in a very short time, with questionable longterm benefit. Many patients relapse.

Xiang Qian: With all due respect, based on personal experience, one cannot conclude that ketamine has no long-lasting benefits for pain patients. There are extensive high quality data to support ketamine's benefit for both acute perioperative and chronic pain. The articles cited [3-5] are interesting but data is scarce to support 
the cancer-promoting side effects of ketamine; there is a lot of more data, in contrast, suggesting that opioids and volatile anesthetics may promote cancer spread. Ketamine has gained momentum for its use in palliative care settings and has an irreplaceable role for treating pain in certain cancer patients. After all, we are discussing benefits and risks. Nothing is perfect.

Lingzhong Meng: It is indeed essential to mentally probe the pros and cons of this bogeyman. Therefore, medicine is not math. Neither is science, probably. It is more or less a philosophy.

Jin Jun Luo: I agree that we need to see more evidence. We have learned that interventions initially thought to be beneficial, such as clinically administrating estrogen for preventing dementia in menopausal women, long chain unsaturated fatty acids for atherosclerosis, neurotrophic factors for nerve regeneration, steroids for Guillain-Barre syndrome, and steroids for spinal traumatic injury, have been proven to be ineffective and sometimes harmful. Accordingly, clinical guidelines have been modified to guide management. Practically, any intervention, even praying, may work in some patients no matter if you believe in it or not. There are articles supporting this effect in prestigious journals. I believe psychological assurance also plays an important role. We may easily see these effects clinically and in the literature from many clinical trials.

Xiang Qian: I fully support evidence-based practice, and I also agree with Dr. Luo and Dr. Meng that we need to be cautious about the side effects and perhaps psychological placebo effects of ketamine.

Jin Jun Luo: I am not against ketamine use for acute pain as I do not have experience with it, but I do see a group of patients with chronic pain without significantly long-term effects except a big bill.

Xiang Qian: At this time, data on the use of ketamine in treating chronic pain is still controversial. The best data are for chronic regional pain syndromes. The point is that we routinely use ketamine for both acute and chronic pain patients; if you know how to dose it properly, and if you select for patients meeting appropriate criteria (e.g. rule out psychological comorbidities), ketamine could really help our patients. We have patients coming from all over the country, especially this year and the year before.

Lingzhong Meng: The hard lesson we learned is from the perioperative use of beta-blockers. At one point, its use almost became standard and mandatory. Now, we realize that it is good for the heart, but maybe bad for the brain. The same caution should be applied to cancer patients who are chronically using ketamine.

Jin Jun Luo: We stopped using ketamine for chronic regional pain syndrome (CRPS) in our department several years ago due to disappointing results. I also saw many patients with CRPS in my clinic. Some of them received ketamine intravenous infusions with short-term effects, which is a far cry from being painfree in the long term. I believe that every disease has a cause and that this etiology should be treated first. In my practice, many patients came to see me with "neuropathy". I strongly discourage simply using a pain medication, such as gabapentin, to treat patients without looking into the cause, although it is not wrong to do so as it is recommended in the "guidelines". I would recommend searching for the underlying medical conditions to elucidate and then treat the etiology. We know that some etiologies of pain may be labeled "idiopathic", but are really in part due to sub-optimal investigation. Chronic pain management is a significant clinical challenge.

Xiang Qian: I agree with you. We should always try to first identify and treat the underlying etiology. There is no question about it.

Jin Jun Luo: Usually, I spend time talking with patients and provide them with reading materials. I encourage patients to work to minimize unnecessary medication, engage in family and social activities, control body weights, drink more water, and engage in adequate physical activities, especially swimming if possible. I also warn them that their pain may initially worsen but will subsequently improve. I then observe the patients in 3-4 months of follow-up. This method works in some patients. I do not like the term fibromyalgia because I think it is misleading. I wrote an editorial comment stating this syndrome may be due to increased central excitability. It may also be related to other chronic pain conditions, including 
CRPS. Therefore, the administration of a tricyclic antidepressant may be a better choice than peripheral manipulation. I may be wrong, but I do have several cases with good results. Talking to patients is very important to help them understand their prognosis. Patients often have exaggerated expectations of the effects of pharmacology.

Xiulu Ruan: I am not sure if you have heard the story of the well-known anesthesiologist Dr. Scott Reuben, a pioneer researcher in preoperative pain control and multimodal analgesia who published many fraudulent papers relating to drugs such as Celebrex, Vioxx, and Lyrica. Anesthesia \& Analgesia published 10 of his articles. His case is considered the biggest in the history of anesthesiology and he is considered the medical equivalent of Bernie Madoff!

Weidong Gao: Yes. Who found out that he was making up data?

Xiulu Ruan: The point that Dr. Luo is making is that we have to be critical of publications and see if there are any conflicts of interest rather than just following the recommendations of a publication. It is impossible for one to publish two dozen randomized placebo controlled trials in a couple of years. It was found that did not have IRB approvals for many of the trials he claimed he had done; they were simply fabricated.

Weidong Gao: That is right. Even the prestigious NEJM recommended hormone therapy for menopausal women, which is now pretty much considered wrong and is abandoned. Am I right?

Xiulu Ruan: I heard that the day before Anesthesia \& Analgesia's announcement to withdraw all of Dr. Reuben's papers, speakers were still talking about and praising his work at a conference. Some in the audience had already learned of the decision to withdraw those papers. Sad.

Xiulu Ruan: He was too extraordinary so he got caught. I'm sure he is not the only one!

Xiang Qian: His work was fabricated but this does not mean that multimodal pain control should be labeled as false science. He was just one of hundreds of researchers working on similar topics but rose to the top in a short time due to his publication record.

Xiulu Ruan: I agree. I use combinational pharmacotherapy or a multimodal approach in treating my chronic pain patients all the time. Even though my clinic is an interventional pain practice, a comprehensive approach is necessary. I think that the best approach should be individualized and comprehensive. However, one has to pay special attention to pharmaceutical company-sponsored research because it is more likely than not to be biased, rendering a conclusion favorable to the sponsoring party. This is not hard to understand.

Peishan Zhao (Tuft Medical Center, USA): Is there any study of ketamine use in the obstetric population? I know people use it to supplement inadequate regional anesthesia.

Yuying Tang (Sichuan University, China): In our hospital, ketamine has been widely used in obstetric anesthesia and during pediatric cardiovascular interventional surgery. A dose of $0.5-1 \mathrm{mg} / \mathrm{kg}$ usually has no obvious clinical side effect on the fetus. Years ago, I saw a cesarean section case in which the patient had an inadequate intrathecal block. However, the case was successfully performed with intravenous ketamine administration. In patients with preeclampsia, ketamine has been used with propofol during anesthesia induction. Blood pressure seemed to be stable. Nowadays, ketamine has gradually been replaced by remifentanil in obstetric anesthesia. However, compared with remifentanil, the potential benefit of ketamine for purpura with hemorrhagic shock and fetal distress deserves to be explored.

\section{Dajin Sun (Shanghai Jiao Tong University, Chi-}

na): I suffered from forehead blisters and severe pain due to herpes zoster of the first branch of the left trigeminal nerve on Feb 2, 2010. The pain caused by herpes zoster felt like being cut with a knife and was very intolerable. I tried all kinds of analgesic treatments such as morphine, fentanyl transdermal patches, oral gabapentin and pregabalin, local nerve block, and acupuncture bloodletting of the fingers and scalp, but they were all useless. Based on my own years of clinical experience, I requested to be hospitalized and treated with ketamine intravenously. The treatment took effect immediately at the dose of 40- 
$60 \mathrm{mg}$. The pain was relieved after one week, and I was discharged from the hospital on March 16. I want to thank my therapy team for their hard work and receptiveness to my suggestions. Ketamine's analgesic effects in treating herpes zoster pain outweigh its side effects such as hallucination. Moreover, ketamine has less suppression on respiration and circulation. The dose used to treat pain is much less than that for surgical anesthesia. It is unnecessary to worry about addiction.

\section{$\underline{\text { Summary }}$}

Ketamine, a phencyclidine hydrochloride derivative, is a noncompetitive antagonist of the N-methyl-D-aspartate subtype of excitatory amino acid receptors. It has unique pharmacological properties and a wide range of clinical applications. However, the long-term effects of ketamine use in various patient populations need to be evaluated in large-scale and outcome-oriented studies. Therefore, before putting more restrictions on ketamine use, a wide range of discussion and more meaningful research should be conducted to define its role in benefiting various patient populations. At the same time, efforts to contain its abuse should be reinforced.

\section{Competing interests}

The authors declare no competing interests.

\section{Acknowledgments}

This review article was written without the support of any funding.

\section{Address for correspondence:}

Lingzhong Meng, MD, Department of Anesthesia and Perioperative Care, University of California San Francisco, 500 Parnassus Avenue, San Francisco, California 94143.

Lingzhong.Meng@ucsf.edu. 415-502-2297 (Phone).

\section{Editor}

Yuguang Huang,MD

Professor and Chairman

Department of Anesthesiology

Peking Union Medical College Hospital

Beijing 100730

garybeijing@163.com

\section{Disclosure of Funding}

None
Additional publication details

Journal short name: Transl Perioper \& Pain Med

Received Date:June 20, 2015

Accepted Date: July 15, 2015

Published Date: July 30 15, 2015

Transl Perioper \& Pain Med 2015; 2(2):20-26

Citation and Copyright

Citation: Meng L, Li J, Lu Y, Sun D, Tao Y, Liu R, Luo J. Ketamine - A Multifaceted Drug. Transl Perioper \& Pain Med 2015; 2(2): $20-26$

Copyright: (C) 2015 Lingzhong Meng, MD, et al. This is an open-access article distributed under the terms of the Creative Commons Attribution License, which permits unrestricted use, distribution, and reproduction in any medium, provided the original author and source are credited.

\section{References}

1. Krystal JH, Karper LP, Seibyl JP, et al. Subanesthetic effects of the noncompetitive NMDA antagonist, ketamine, in humans. Psychotomimetic, perceptual, cognitive, and neuroendocrine responses. Arch Gen Psychiatry. 1994;51(3):199214.

2. Krystal JH, Petrakis IL, Webb E, et al. Dose-related ethanol-like effects of the NMDA antagonist, ketamine, in recently detoxified alcoholics. Arch Gen Psychiatry. 1998;55(4):354-60.

3. Shapiro J, Jersky J, Katzav S, et al. Anesthetic drugs accelerate the progression of postoperative metastases of mouse tumors. J Clin Invest. 1981;68:678-685.

4. Katzav S, Shapiro J, Segal S, et al. General anesthesia during excision of a mouse tumor accelerates postsurgical growth of metastases by suppression of natural killer cell activity. Isr J Med Sci. 1986;22:339-345.

5. Melamed R, Bar-Yosef S, Shakhar G, et al. Suppression of natural killer cell activity and promotion of tumor metastasis by ketamine, thiopental, and halothane, but not by propofol: mediating mechanisms and prophylactic measures. Anesth Analg. 2003;97:1331-1339.

6. Sacerdote P. Opioid-induced immunosuppression. Curr Opin Support Palliat Care. 2008; 2(1):14-8.

7. Vallejo R, de Leon-Casasola O, Benyamin R. Opioid therapy and immunosuppression: a review. Am J Ther. 2004;11(5):354-65. 
8. Murrough JW, Iosifescu DV, Chang LC, Al Jurdi RK, Green CE, Perez AM, Iqbal S, Pillemer S, Foulkes A, Shah A, Charney DS, Mathew SJ. Antidepressant efficacy of ketamine in treatment-resistant major depression: a two-site randomized controlled trial. Am J Psychiatry. 2013 Oct;170(10):1134-42.

9. McGirr A, Berlim MT, Bond DJ, Fleck MP, Yatham LN, Lam RW. A systematic review and meta-analysis of randomized, double-blind, placebo-controlled trials of ketamine in the rapid treatment of major depressive episodes. Psychol Med. 2015 Mar;45(4):693-704. 